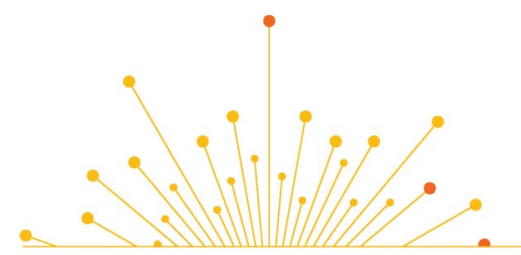

SCIENCE TABLE

COVID-19 ADVISORY FOR ONTARIO

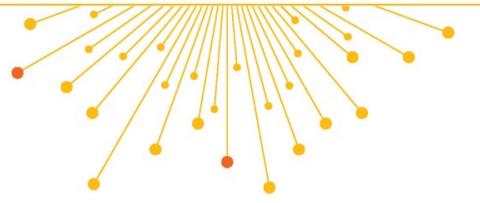

Version 1.0

Published: March 23, 2021

Citation: Kain D, Stall NM, Allen V, et al. Routine asymptomatic SARS-CoV-2 screen testing of Ontario long-term care staff after COVID-19 vaccination. Science Briefs of the Ontario COVID -19 Science Advisory Table. 2021;2(15). https:// doi.org/10.47326/ocsat.2021.02.15.1.0

Author Affiliations: The affiliations of the members of the Ontario COVID-19 Science Advisory Table can be found at https:// covid19-sciencetable.ca/.

Declarations of Interest: The declarations of interest of the members of the Ontario COVID-19 Science Advisory Table, its Working Groups, or its partners can be found at https://covid19-sciencetable.ca/. The declarations of interest of external authors can be found under additional resources at https:// doi.org/10.47326/ocsat.2021.02.15.1.0.

About Us: The Ontario COVID-19 Science Advisory Table is a group of scientific experts and health system leaders who evaluate and report on emerging evidence relevant to the COVID-19 pandemic, to inform Ontario's response. Our mandate is to provide weekly summaries of relevant scientific evidence for the COVID-19 Health Coordination Table of the Province of Ontario, integrating information from existing scientific tables, Ontario's universities and agencies, and the best global evidence. The Science Table summarizes its findings for the Health Coordination Table and the public in Science Briefs.

The Congregate Care Setting Working Group is a group of internationally recognized researchers with expertise in older people living in congregate care settings. The Working Group evaluates emerging scientific evidence related to congregate care settings to inform Ontario's response to the COVID-19 pandemic. The Working Group reports its findings to the public and the Science Table. Its findings are also summarized in Science Briefs.

Correspondence to: Secretariat of the Ontario COVID-19 Science Advisory Table (info@covid19-sciencetable.ca)

Copyright: 2021 Ontario COVID-19 Science Advisory Table. This is an open access document distributed under the terms of the

\section{Routine Asymptomatic SARS-CoV-2 Screen Testing of Ontario Long-Term Care Staff After COVID-19 Vaccination}

Dylan Kain, Nathan M. Stall, Vanessa Allen, Gerald A. Evans, Jessica Hopkins, Fiona G. Kouyoumdjian, Allison McGeer, Samira Mubareka, Samir N. Patel, Paula A. Rochon, Brian Schwartz, Peter Jüni, Jennie Johnstone on behalf of the Congregate Care Setting Working Group and the Ontario COVID-19 Science Advisory Table

\section{Key Message}

SARS-CoV-2 screen testing is routine serial testing of asymptomatic individuals outside of outbreak or known exposure settings to identify staff infectious with SARS -CoV-2 and exclude them from work. Routine asymptomatic screen testing of staff has been proposed as a potential mitigating strategy to reduce SARS-CoV-2 introduction and transmission in long-term care (LTC) homes.

A rapid review of the literature found no real-world evidence to either support or refute screen testing in preventing LTC home COVID-19 outbreaks. There are several direct harms associated with screen testing, as well as opportunity costs, including exacerbating LTC staffing shortages.

On the basis of the evidence reviewed, and given the high rates of protection of COVID-19 vaccines against symptomatic and asymptomatic SARS-CoV-2 infection, the potential harms and costs of screen testing among vaccinated LTC home staff likely outweigh the benefits.

\section{Summary}

\section{Background}

COVID-19 has had a devastating impact on Ontario's LTC homes, with residents of these homes at disproportionately high risk of both contracting SARS-CoV-2 and experiencing poor COVID-19 outcomes. Many LTC home outbreaks begin when SARS -CoV-2 is imported from the community by staff who reside in neighbourhoods with high SARS-CoV-2 incidence. As of July 1, 2020, the Ontario Ministry of Long-Term Care instructed all LTC home staff to undergo routine asymptomatic SARS-CoV-2 screen testing in an effort to prevent COVID-19 outbreaks.

\section{Questions}

What is the potential benefit and evidence for routine asymptomatic SARS-CoV-2 screen testing of LTC staff in preventing LTC home COVID-19 outbreaks?

What are the potential harms of routine asymptomatic SARS-CoV-2 screen testing of LTC staff?

What are the opportunity costs and implementation challenges of routine asymptomatic SARS-CoV-2 screen testing of LTC staff?

How does COVID-19 vaccination of LTC staff and residents impact the need for ongoing asymptomatic SARS-CoV-2 screen testing of staff? 
Creative Commons Attribution License, which permits unrestricted use, distribution, and reproduction in any medium, provided that the original work is properly cited.

The views and findings expressed in this Science Brief are those of the authors and do not necessarily reflect the views of all of the members of the Ontario COVID-19 Science Advisory Table, its Working Groups, and its partners.

\section{Findings}

There are theoretical benefits to routine asymptomatic screen testing in LTC home staff. These include identifying asymptomatic or pre-symptomatic SARS-CoV-2 infections to reduce potential importation into homes, identifying symptomatic cases that may have been missed in symptom screening programs, and identifying outbreaks at an earlier stage or that may have been missed due to breakdown in other surveillance systems. Despite these theoretical benefits, a rapid review reveals no real-world evidence to either support or refute the effectiveness of routine asymptomatic screen testing of LTC staff in preventing LTC home COVID-19 outbreaks.

Between June 28, 2020 and March 13, 2021, a total of 705,370 SARS-CoV-2 tests were performed on asymptomatic Ontario LTC staff which yielded 1,147 positive results $(0.16 \%$ test positivity). Overall test positivity rates ranged from $<0.1 \%$ during times of low SARS-CoV-2 incidence (weekly incidence rate $<10$ per 100,000) across the Province of Ontario, increasing to a peak of $0.36 \%$ during times of very high incidence (weekly incidence rate $\geq 40$ per 100,000 ).

Potential harms of routine asymptomatic screen testing include: 1) physical discomfort and injury from frequent nasopharyngeal swabbing; 2) staff behaviour change associated with knowledge that screen testing is negative and 3) false positive results, especially when testing is performed in the setting of low SARS-CoV-2 incidence (low pre-test probability). The opportunity costs of RT-PCR screen testing include increasing provincial laboratory test turn-around times, especially during times of high demand for SARS-CoV-2 testing and in the context of limited laboratory capacity. While rapid antigen tests have the benefit of being less expensive and providing rapid results, due to lower test sensitivity they require more frequent testing and thus substantial increases in health human resources which may exacerbate LTC staffing shortages.

COVID-19 vaccines demonstrate high rates of protection against SARS-CoV-2 infection and there is emerging evidence for prevention of transmission. As of March 21, 2021, over 68,000 residents (98\%) and over 73,000 staff $(74 \%)$ in Ontario LTC homes have received at least one dose of mRNA vaccine. Eight weeks after the start of vaccination, the estimated relative reduction in SARS-CoV-2 incidence was $89 \%$ in LTC residents and $79 \%$ in LTC staff. The estimated relative reduction in COVID-19 deaths in LTC residents was $96 \%$ after 8 weeks.

\section{Interpretation}

There is no available real-world evidence to either support or refute the benefit of routine asymptomatic screen testing in preventing COVID-19 outbreaks. Screen testing among Ontario LTC staff between June 28, 2020 and March 13, 2021 yielded an overall test positivity of $0.16 \%$, which decreased to $<0.1 \%$ during periods of low SARS-CoV-2 community incidence. Given the high rates of protection of COVID-19 vaccines against SARS-CoV-2 infection and emerging evidence for prevention of transmission, asymptomatic routine screen testing is likely of low yield in vaccinated staff outside of an exposure, or outbreak setting. The potential harms and opportunity costs likely outweigh the benefits of ongoing screen testing among vaccinated LTC home staff, even when B.1.1.7 is the dominant SARS-CoV-2 variant.

\section{Background}

In Ontario there are 626 LTC homes with 69,799 residents occupying 77,257 longstay beds as of January $2021 .{ }^{1}$ Ontario's LTC homes employ approximately 100,000 
clinical and non-clinical staff, who are involved in clinical work, caregiving, administration, housekeeping, food preparation, facilities management, maintenance, and recreation. ${ }^{2}$ LTC homes were the epicenter of Ontario's COVID-19 pandemic. As of March 11, 2021, ${ }^{3}$ there have been a total of 14,976 SARS-CoV-2 infections and 3,749 COVID-19 deaths in LTC residents, as well as 6,703 SARS-CoV-2 infections and 11 COVID-19 deaths in LTC staff.

The most important predictor of whether a LTC home will experience a COVID-19 outbreak is the daily incidence of SARS-CoV-2 infections in the communities surrounding the homes. ${ }^{4}$ Many LTC home outbreaks begin when SARS-CoV-2 is imported from the community by staff who reside in neighbourhoods with high SARS-CoV-2 incidence. This risk of importation is exacerbated by the absence of universal paid sick leave, employment of part-time staff who work multiple frontfacing jobs, and staff who are still able to work in multiple healthcare settings concurrently. ${ }^{1,5}$

Multiple interventions have been implemented in Ontario LTC homes to both reduce importation of SARS-CoV-2 infection into homes and transmission within LTC homes. This includes increased staffing, hiring of infection prevention and control (IPAC) staff, IPAC training for LTC staff, better supply of personal protective equipment (PPE), universal masking and face shield use, enhanced symptom screening for LTC staff and residents, improved access to SARS-CoV-2 testing and shorter turn-around times for processing and reporting results, reduced crowding, limiting most staff from working in more than one healthcare setting within a 14-day period, and hospital partnerships to improve IPAC. . $^{1,6-8}$

Routine asymptomatic SARS-CoV-2 screen testing of staff was also implemented as a potential mitigating strategy to reduce SARS-CoV-2 importation and transmission in LTC homes. Screen testing is routine serial testing of asymptomatic individuals outside of outbreak or known exposure settings to identify staff infectious with SARS -CoV-2 and exclude them from work.

Screen testing of LTC staff was initiated on May $31,2020,{ }^{9}$ when all Ontario LTC staff were instructed to submit samples for serial RT-PCR SARS-CoV-2 testing twice per month, and this was formalized into policy on July $1,2020 .^{10}$

This policy was revised on November 22, 2020, ${ }^{11}$ with LTC staff screen testing increasing to once a week in high SARS-CoV-2 incidence areas (orange, red and grey zones with weekly incidence rates of $\geq 25$ per 100,000 ) and every two weeks in low incidence areas (green and yellow zones with weekly incidence rates of $<25$ per $100,000)$ in accordance with Ontario's colour-coded COVID-19 response framework. ${ }^{12}$ In January 2021, the Ministry of Long-Term Care asked LTC homes to begin shifting screen testing of staff from RT-PCR to rapid antigen testing, with plans to complete the transition by March $15,2021 .^{13}$

\section{Questions}

What is the potential benefit and evidence for routine asymptomatic SARS-CoV-2 screen testing of LTC staff in preventing LTC home COVID-19 outbreaks?

What are the potential harms of routine asymptomatic SARS-CoV-2 screen testing of LTC staff?

What are the opportunity costs and implementation challenges of routine asymptomatic SARS-CoV-2 screen testing of LTC staff?

How does COVID-19 vaccination of LTC staff and residents impact the need for ongoing asymptomatic SARS-CoV-2 screen testing of staff? 


\section{Findings}

\section{Potential Benefits of and Evidence for Screen Testing}

The potential benefits of routine asymptomatic SARS-CoV-2 screen testing of LTC staff include: 1 ) detecting asymptomatic or pre-symptomatic staff with SARS-CoV-2 infection to prevent importation into LTC homes; 2) case finding of very mildly symptomatic staff who misattributed symptoms to other causes or who may be reluctant to stay home from work when ill because of the absence of universal paid sick leave; and 3) functioning as a safety net to alert the LTC home, public health units (PHUs) or hospital partners of a developing outbreak if not identified by other surveillance systems.

A jurisdictional scan of provincial LTC home screen testing practices was completed in February and March 2021. Ontario has the most extensive routine asymptomatic staff screen testing program in Canada. Quebec has the second most extensive program consisting of once weekly RT-PCR testing of LTC staff in high incidence areas (Johnstone J, personal communication). Alberta has announced a plan to initiate a voluntary rapid antigen testing program for LTC staff in homes not in outbreak. ${ }^{14}$ British Columbia ${ }^{15}$ and Manitoba ${ }^{16}$ have pilot projects involving rapid antigen testing in a few select LTC homes, but without current plans for more widespread surveillance testing. Saskatchewan, Prince Edward Island, and Newfoundland have no routine screen testing programs. Nova Scotia and New Brunswick are conducting sentinel surveillance in LTC staff on a voluntary basis by RT -PCR (Johnstone J, personal communication). ${ }^{17}$

A rapid review by Ontario's Evidence Synthesis Network revealed no real-world evidence to either support or refute the effectiveness of routine asymptomatic SARS -CoV-2 screen testing of LTC staff in preventing LTC home COVID-19 outbreaks as of March 11, 2021. ${ }^{18}$

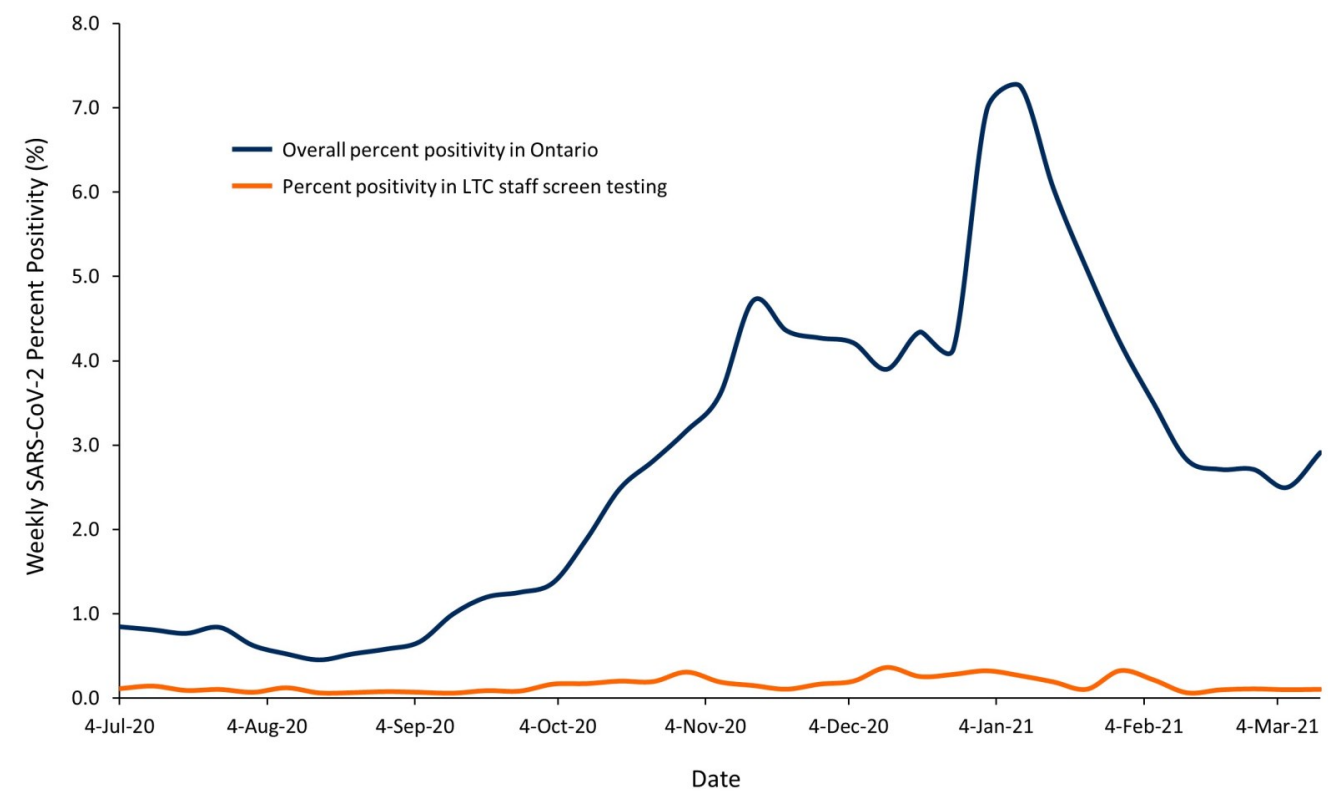

Figure 1. Ontario LTC Staff Screen Testing SARS-CoV-2 Percent Positivity Compared to Provincial Percent Positivity, from June 28, 2020 to March 13, 2021

Line graph showing percent SARS-CoV-2 test positivity for routine asymptomatic LTC staff screen testing compared to provincial test positivity between June 28, 2020 and March 13, 2021. During the low SARS-CoV-2 incidence summer months of 2020, test positivity rates were consistently $<0.1 \%$. There was a small increase in SARS-CoV-2 test positivity during the second wave (September 1, 2020-February 28, 2021) peaking at 0.36\% in early January 2021. LTC, long-term care. 
In Ontario, a total of 705,370 SARS-CoV-2 tests were performed on asymptomatic LTC staff between June 28, 2020 and March 13, 2021 which yielded 1,147 positive results $(0.16 \%$ test positivity). Overall test positivity rates ranged from $<0.1 \%$ during times of low SARS-CoV-2 incidence (weekly incidence rate $<10$ per 100,000, in accordance with Ontario's colour-coded COVID-19 response framework) across the Province of Ontario, increasing to a peak of $0.36 \%$ during times of very high incidence (weekly incidence rate $\geq 40$ per 100,000, in accordance with Ontario's colour-coded COVID-19 response framework) ${ }^{12}$ (Figure 1). These Ontario findings align with multiple Canadian and international studies demonstrating a $<0.5 \%$ positivity rate in asymptomatic individuals tested for SARS-CoV-2 in low incidence settings (COVID-19 Provincial Diagnostic Network Laboratories, personal communication). ${ }^{19-22}$

\section{Potential Harms of Screen Testing}

There are several potential harms of routine asymptomatic SARS-CoV-2 screen testing of LTC staff. Direct harms include the pain and discomfort of repeated nasopharyngeal testing, which on very rare occasions can result in more serious injury including serious bleeding and infection. ${ }^{23,24}$ Repeated testing may also result in testing fatigue, meaning that symptomatic staff may delay SARS-CoV-2 testing until their next regularly scheduled testing. For example, if a staff becomes symptomatic on a Saturday, but has a scheduled test for the coming Monday, they could choose to avoid the inconvenience of an extra trip to get tested as soon as possible. Repeated testing also risks exacerbating existing LTC staffing shortages, with anecdotal reports of staff leaving or considering exiting the sector, especially as they are not consistently paid for time to get tested.

Another harm is the potential for behaviour change associated with knowledge of negative test results, resulting in lower compliance with recommended public health interventions. ${ }^{25}$ This has been suggested as a driver in the White House Rose Garden COVID-19 outbreak in the fall of 2020, where negative SARS-CoV-2 test results were reported to contribute to a feeling of safety, and non-compliance with masking and physical distancing. ${ }^{26}$

Routine asymptomatic staff screen testing also carries the risk of false positive results. While the analytical specificity of SARS-CoV-2 testing by RT-PCR is nearly $100 \%$, there are several reasons why the real-world specificity is lower, often estimated to be around 99.9 to $99.99 \% .{ }^{27,28}$ Reasons include mis-labelled specimens, and contamination in laboratories.

In situations with a very low pre-test probability, the positive predictive value of SARS-CoV-2 by RT-PCR will be poor despite a high test specificity. Assuming the pretest probability for routine asymptomatic screen testing of LTC staff to be $0.1 \%$ (the rate of positivity in Ontario LTC staff during times of low SARS-CoV-2 incidence) and using a test sensitivity of $90 \%$ and specificity of $99.95 \%$, the positive predictive value of such testing is only $64 \%$, while the false positive rate is $36 \%$. This means that 36 out of 100 positive tests are falsely positive. In contrast, assuming the pre-test probability for a SARS-CoV-2 test in a symptomatic individual is $10 \%$, the positive predictive value is $99.5 \%$ while the false positive rate is $0.5 \%$.

Each of these false positive tests has direct negative consequences on LTC staff and residents. Staff with false positive test results are removed from the workforce, while other staff linked to those testing positive may also be excluded from work, which together can exacerbate staffing shortages. False positive test results also have other downstream consequences requiring resources and time of LTC homes, public health investigators, and hospital partners to manage suspected COVID-19 
outbreaks. False positive results may also result in unnecessary testing of LTC residents and placement in droplet/contact precautions for 14 days due to the perceived exposure. The latter can worsen loneliness and psychological distress among residents, and potentially result in increased use of psychotropic medications. ${ }^{29}$ Further unnecessary testing of residents may be required as well.

\section{Opportunity Costs and Implementation Challenges of Screen Testing}

The large daily volume of routine asymptomatic SARS-CoV-2 screen testing for LTC home staff when done by RT-PCR also occupies a substantial fraction of the daily provincial testing capacity. In times of high SARS-CoV-2 testing demand, this can result in slower test turn-around times in other settings. Furthermore, a slow test turn-around time can also render the screen test useless if an infected LTC staff continues to work because of a delay in receiving a positive test result. In early October 2020 , only $5.5 \%$ of routine asymptomatic staff screen tests in Ontario were reported within 24 hours and only 32\% within 48 hours (Figure 2). This has, however, substantially improved in recent months.

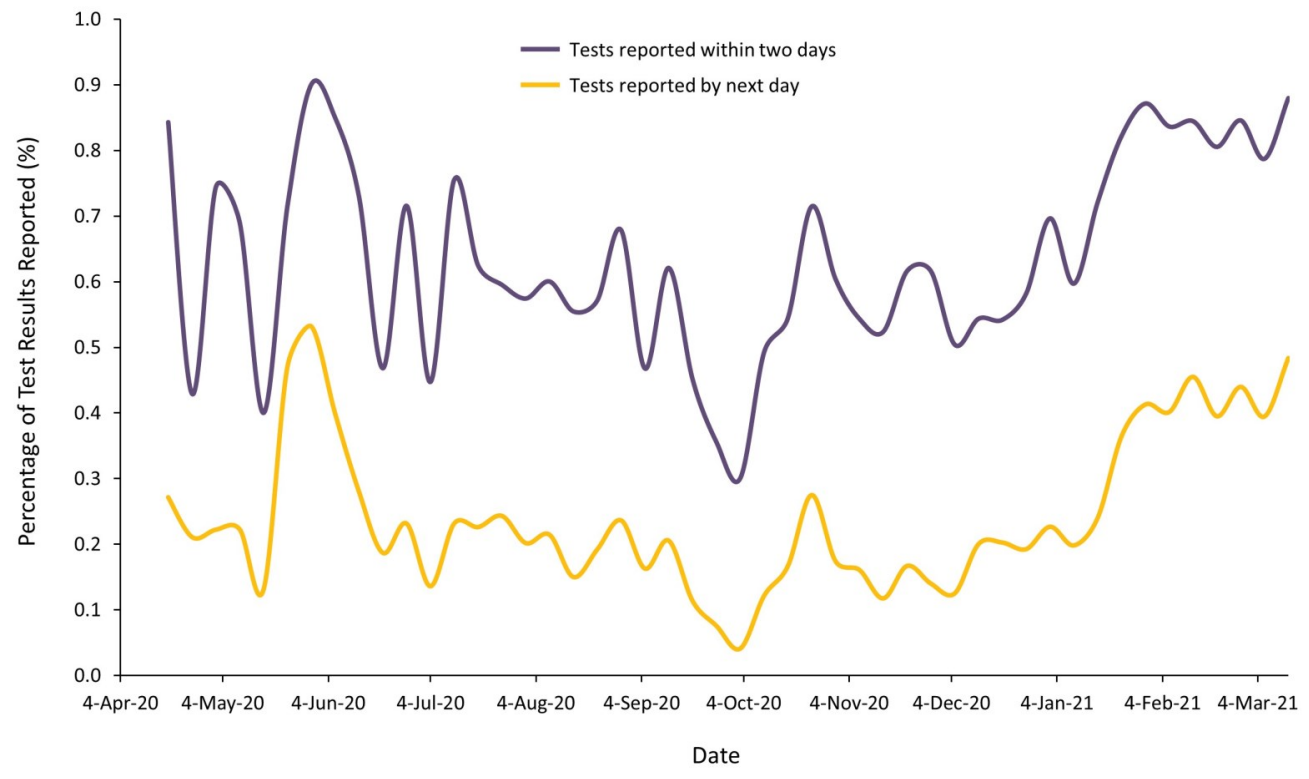

Figure 2. RT-PCR Test Turn-Around Times for Screen Testing of Ontario LTC Staff, from April 12, 2020 to March 13, 2021 Line graph illustrating asymptomatic routine LTC staff screen test turn-around times in Ontario. The purple line represents the percentage of all RT-PCR tests that were reported within 48 hours of collection and the yellow line is the percentage of all tests that were reported within 24 hours of collection. LTC, long-term care.

In January 2021, the Ontario Ministry of Long-Term Care asked LTC homes to begin shifting screen testing from RT-PCR to rapid antigen testing, with plans to complete the transition by March 15, 2021. ${ }^{13}$ Rapid antigen testing has the benefits of being much less expensive than RT-PCR and more comfortable as it is more commonly collected with a combined swab of throat and both nares or a deep nasal swab rather than with a nasopharyngeal swab. ${ }^{13}$ Additionally, by providing nearly immediate results, rapid antigen testing can address the issue of test turn-around times. This comes, however, at the cost of lower test sensitivity, ${ }^{30}$ requiring an increased testing frequency of 2-3 times per week in high SARS-CoV-2 incidence regions and once per week in low SARS-CoV-2 incidence regions. ${ }^{31,32}$ The LTC staff resources needed to operationalize the rapid antigen testing strategy is much higher than with RT-PCR, with estimates of each home requiring an additional two full-time employees required for implementation. ${ }^{33}$ Thus the reduced costs of rapid antigen testing associated with eliminating laboratory needs is offset by much higher health human resource costs in LTC homes. Ontario's proposed transition to rapid antigen testing comes at a time when staff shortages within the LTC sector are already 
extremely high, with serious concerns about whether homes will be able to hire new employees, or whether this strategy will draw from existing strained resources, and thus potentially negatively affect resident care.

The practicality of thrice weekly rapid antigen testing is also challenging, as it takes roughly 20-30 minutes for the entire testing process, and sufficient time and space are needed to allow staff to maintain physical distance from each other while tests are being processed to avoid SARS-CoV-2 transmission. Time spent undergoing testing may reduce time spent with residents, which may further compromise LTC staffing and care.

\section{Considerations for Screen Testing After COVID-19 Vaccination}

The Pfizer-BioNTech and Moderna COVID-19 vaccines have demonstrated extremely high efficacy in preventing symptomatic COVID-19 infection at 95\% and 94\%, respectively. ${ }^{34,35}$ As of March 21, 2021, over 68,000 Ontario LTC residents received at least one dose of one of these COVID-19 vaccines (98\%) with over 61,000 having received both doses. Over 73,000 Ontario LTC staff have also received at least one dose of a COVID-19 vaccine (74\%), with over 49,000 having received both doses. Eight weeks after the rollout of COVID-19 vaccines to Ontario LTC homes, the estimated relative reduction in SARS-CoV-2 incidence was $89 \%$ in LTC residents and $79 \%$ in LTC health care workers. Further, the estimated relative reduction in COVID19 mortality in LTC residents was $96 \%$ after 8 weeks. ${ }^{36}$

There is also growing evidence suggesting that COVID-19 vaccines also reduce asymptomatic SARS-CoV-2 infections, ${ }^{37-43}$ and can reduce transmission. For example, the recently published SIREN study, in the United Kingdom, showed an $86 \%$ reduction in asymptomatic disease in systematically screened healthcare workers following vaccination, despite the B.1.1.7 variant being the dominant circulating SARS-CoV-2 strain. $^{37}$

In light of this evidence, the likelihood of vaccinated LTC staff testing positive during routine asymptomatic SARS-CoV-2 screen testing is very low. This low pre-test probability means that the positive predictive value of SARS-CoV-2 screen testing is poor, and similar to testing completed in Ontario during periods of low SARS-CoV-2 incidence, the majority of positive results are likely to be false positive results.

\section{Interpretation}

Many LTC home outbreaks begin when SARS-CoV-2 is introduced from the community by asymptomatic or pre-symptomatic staff. To help mitigate this, Ontario has required all LTC staff to undergo routine asymptomatic SARS-CoV-2 screen testing since May 31, 2020. This requirement was formalized on July 1, 2020, and since July, Ontario has completed over 760,000 routine asymptomatic LTC staff screen testing for SARS-CoV-2 tests by RT-PCR.

Despite the theoretical benefits of routine asymptomatic SARS-CoV-2 screen testing, including detection of pre-symptomatic, asymptomatic, or very mildly symptomatic staff infected with SARS-CoV-2, and detection of COVID-19 outbreaks when not identified by other surveillance systems, there is no available real-world evidence to support or refute the benefit of screen testing in preventing LTC home COVID-19 outbreaks. Ontario is the only Canadian province requiring universal mandatory screen testing among all LTC home staff, and testing completed between June 28, 2020 and March 13, 2021 yielded an overall test positivity of $0.16 \%$, which decreased to $<0.1 \%$ during periods of low SARS-CoV- 2 community incidence. This analysis is limited by incomplete capture of all asymptomatic SARS-CoV-2 screen test 
results among Ontario LTC staff. However, the results are consistent with other asymptomatic testing campaigns. ${ }^{19-22}$

There are several potential harms of routine asymptomatic screen testing including staff physical discomfort and injury from frequent nasopharyngeal swabbing, staff behaviour change associated with knowledge that screen testing is negative, and a high rate of false positive results which can exacerbate staffing shortages and cause unnecessary testing and isolation of staff and residents. Universal staff screen testing can also impact provincial laboratory testing capacity and test turn-around times.

A planned transition from RT-PCR to rapid antigen testing to support routine asymptomatic screen staff testing across Ontario LTC homes may reduce inlaboratory testing costs and alleviate pressures on provincial microbiology laboratories, but has substantial implementation challenges, including significant increases in requirements for staffing to operate and oversee testing, and risks associated with false positive and false negative tests.

With more than $95 \%$ of LTC residents and over $70 \%$ of all LTC staff in Ontario having received at least one dose of a COVID-19 vaccine, and real-world evidence for both protection against symptomatic and asymptomatic SARS-CoV-2 infection, ongoing province-wide screen testing in COVID-19 vaccinated staff is likely low yield, especially in the absence of known exposures and outside of outbreak settings, even when B.1.1.7 is the dominant SARS-CoV-2 variant. ${ }^{44}$ The low yield of asymptomatic SARS-CoV-2 screen testing likely extends to essential family caregivers who have been vaccinated against COVID-19.

\section{Methods Used for This Science Brief}

A jurisdictional scan of provincial LTC home staff SARS-CoV-2 surveillance testing practices was completed in February and March 2021 through email inquiries to infection control practitioners, infectious disease specialists and geriatricians across the country, as well as by reviewing relevant media stories.

A comprehensive rapid review of the published and grey literature for evidence on the effectiveness of routine asymptomatic SARS-CoV-2 screen testing of health care workers in preventing LTC home COVID-19 outbreaks was completed by Ontario's Evidence Synthesis Network and was last updated as of March 11, 2021. ${ }^{18}$ To identify relevant articles and documents for this review, the following search terms were used in combination: "asymptomatic", "symptomatic", "congregate care", "long term care", "nursing home", "health care workers", "health care professionals", "COVID-19", "coronavirus", "test", "serial test", "routine test", "vaccine", "vaccination".

Data on routine asymptomatic SARS-CoV-2 staff screen testing of Ontario LTC staff was obtained from the Capacity Planning and Analytics Division of the Ontario Ministries of Health and Long-Term Care and was current up until March 13, 2021. All results were reported using descriptive statistics.

\section{Author Contributions}

DK, NMS, and JJ conceived the Science Brief and wrote the first draft. DK performed the analysis. All authors revised the Science Brief critically for important intellectual content and approved the final version. 


\section{References}

1. Stall NM, Brown KA, Maltsev A, et al. COVID-19 and Ontario's long-term care homes (full brief). Sci Briefs Ont COVID-19 Sci Advis Table. 2021;1(7). https:// doi.org/10.47326/ocsat.2021.02.07.1.0

2. Gillese EE. Long-term care staffing study. Ontario.ca. Published July 30, 2020. Accessed March 11, 2021. https://www.ontario.ca/page/long-term-care-staffingstudy

3. Government of Ontario. How Ontario is responding to COVID-19. Ontario.ca. Accessed March 11, 2021. https://www.ontario.ca/page/how-ontario-is-respondingcovid-19\#section-1

4. Malikov K, Huang Q, Shi S, Stall NM, Tuite AR, Hillmer MP. Temporal associations between community incidence of COVID-19 and nursing home outbreaks in Ontario, Canada. J Am Med Dir Assoc. 2021;22(2):260-262. https://doi.org/10.1016/ j.jamda.2020.12.011

5. Jones A, Watts AG, Khan SU, et al. Impact of a public policy restricting staff mobility between long-term care homes in Ontario, Canada during the COVID-19 pandemic. medRxiv. Published online November 18, 2020:2020.11.17.20231498. https://doi.org/10.1101/2020.11.17.20231498

6. Stall NM, Farquharson C, Fan-Lun C, et al. A hospital partnership with a nursing home experiencing a COVID-19 outbreak: description of a multiphase emergency response in Toronto, Canada. J Am Geriatr Soc. 2020;68(7):1376-1381. https:// doi.org/10.1111/jgs.16625

7. The National Collaborating Centre for Methods and Tools. Rapid Review Update 1: What Risk Factors Are Associated with COVID-19 Outbreaks and Mortality in LongTerm Care Facilities and What Strategies Mitigate Risk?; 2020. Accessed March 15, 2021.https://www.nccmt.ca/uploads/media/ media/0001/02/3133bc00b0f5ec21b2efe70a10719e4440493411.pdf

8. Rios P, Radhakrishnan A, Williams C, et al. Preventing the transmission of COVID19 and other coronaviruses in older adults aged 60 years and above living in longterm care: a rapid review. Syst Rev. 2020;9(1):218. https://doi.org/10.1186/s13643020-01486-4

9. Steele R, Anderson M. Re: COVID-19 testing for long-term care home staff. Published online May 31, 2020. Accessed March 11, 2021. https://tchomes.net/ LTCHPORTAL/Content/Snippets/MLTC\%20DM\%20Letter\%20re\%20COVID-19\% 20Testing\%20for\%20Long-Term\%20Care\%20Home\%20Staff_ENM.pdf

10. Government of Ontario. COVID-19 testing for long-term care home staff. Published July 16, 2020. Accessed March 15, 2021. https://www.health.gov.on.ca/ en/pro/programs/Itc/memo_20200716.aspx

11. Steele R. Updated long-term care sector surveillance testing strategy. Published online November 22, 2020. Accessed March 15, 2021. https://ohwestcovid19.ca/wp -content/uploads/2020/11/20201124-DM-Memo-Updated-Long-Term-Care-SectorSurveillance-Testing-Strategy-Nov-22-2020.pdf

12. Government of Ontario. COVID-19 response framework: keeping Ontario safe and open. Ontario.ca. Published November 3, 2020. Accessed March 11, 2021. https://www.ontario.ca/page/covid-19-response-framework-keeping-ontario-safeand-open

13. Government of Ontario. COVID-19: long-term care home surveillance testing and 
access to homes. Ontario.ca. Published February 16, 2021. Accessed March 11, 2021. https://www.ontario.ca/page/covid-19-long-term-care-home-surveillancetesting

14. CBC News. Alberta deploys almost $1 \mathrm{M}$ rapid tests to help screen for COVID-19 | CBC News. CBC. Published March 11, 2021. Accessed March 11, 2021. https:// www.cbc.ca/news/canada/edmonton/hinshaw-covid-vaccines-alberta-1.5945455

15. Zussman R. B.C. is 'duty-bound' to deploy COVID-19 rapid tests in long-term care, industry says. Global News. Published January 19, 2021. Accessed February 23, 2021. https://globalnews.ca/news/7585903/rapid-testing-long-term-care-industry/

16. Government of Manitoba. COVID-19 asymptomatic surveillance testing introduced in three personal care homes. Province of Manitoba. Published December 17, 2020. Accessed February 24, 2021. https://news.gov.mb.ca/news/ index. html?item $=50078$ \&posted $=2020-12-17$

17. Muecke C. COVID-19 Testing and Sentinel Surveillance. New Brunswick Department of Health; 2020. Accessed March 11, 2021. https://813.cupe.ca/ files/2020/09/Bulletin-69e-COVID-19-Testing-and-Sentinel-Surveillance-Sep-32020.pdf

18. Research, Analysis, and Evaluation Branch. Evidence Synthesis Briefing Note: Serial Testing of Asymptomatic Health Care Workers. COVID-19 Evidence Synthesis Network; 2021. https://esnetwork.ca/briefings/serial-testing-of-asymptomatichealth-care-workers-following-vaccination/

19. Cao S, Gan Y, Wang C, et al. Post-lockdown SARS-CoV-2 nucleic acid screening in nearly ten million residents of Wuhan, China. Nat Commun. 2020;11(1):5917. https://doi.org/10.1038/s41467-020-19802-w

20. Snoeck CJ, Vaillant M, Abdelrahman T, et al. Prevalence of SARS-CoV-2 infection in the Luxembourgish population - the CON-VINCE study. medRxiv. Published online May 18, 2020:2020.05.11.20092916. https://doi.org/10.1101/2020.05.11.20092916

21. Olalla J, Correa AM, Martín-Escalante MD, et al. Search for asymptomatic carriers of SARS-CoV-2 in healthcare workers during the pandemic: a Spanish experience. QJM Int J Med. 2020;113(11):794-798. https://doi.org/10.1093/qjmed/ hcaa238

22. Pearson H. COVID-19 asymptomatic tests: 48 positive cases in Calgarians with no known contact with virus. Global News. Published May 19, 2020. Accessed February 24, 2021. https://globalnews.ca/news/6961600/coronavirus-calgary-asymptomatictesting-results/

23. Paquin R, Ryan L, Vale FL, Rutkowski M, Byrd JK. CSF leak after COVID-19 nasopharyngeal swab: a case report. The Laryngoscope. https://doi.org/10.1002/ lary.29462

24. Alberola-Amores FJ, Valdeolivas-Urbelz E, Torregrosa-Ortiz M, Álvarez-Sauco M, Alom-Poveda J. Meningitis due to cerebrospinal fluid leak after nasal swab testing for COVID-19. Eur J Neurol. Published online January 21, 2021. https:// doi.org/10.1111/ene.14736

25. University of Liverpool. Liverpool Covid-19 Community Testing Pilot.; 2020. Accessed March 15, 2021. https://www.liverpool.ac.uk/media/livacuk/coronavirus/ Liverpool,Community, Testing,Pilot,Interim,Evaluation.pdf

26. Fitzpatrick MC, Pandey A, Wells CR, Sah P, Galvani AP. Buyer beware: inflated claims of sensitivity for rapid COVID-19 tests. The Lancet. 2021;397(10268):24-25. https://doi.org/10.1016/S0140-6736(20)32635-0 
27. Guthrie JL, Chen AJ, Budhram DR, et al. Characteristics of SARS-CoV-2 Testing for Rapid Diagnosis of COVID-19 during the Initial Stages of a Global Pandemic. Infectious Diseases (except HIV/AIDS); 2020. https:// doi.org/10.1101/2020.12.23.20231589

28. Public Health Ontario. COVID-19 Laboratory Testing Q\&As.; 2021:6. https:// www.publichealthontario.ca/-/media/documents/lab/covid-19-lab-testing-faq.pdf? la $=$ en

29. Stall NM, Zipursky JS, Rangrej J, et al. Assessment of psychotropic drug prescribing among nursing home residents in Ontario, Canada, during the COVID-19 pandemic. JAMA Intern Med. Published online March 15, 2021. https:// doi.org/10.1001/jamainternmed.2021.0224

30. Dinnes J, Deeks JJ, Adriano A, et al. Rapid, point-of-care antigen and molecularbased tests for diagnosis of SARS-CoV-2 infection. Cochrane Database Syst Rev. 2020;8:CD013705. https://doi.org/10.1002/14651858.CD013705

31. Mina MJ, Parker R, Larremore DB. Rethinking COVID-19 test sensitivity - a strategy for containment. $N$ Engl J Med. 2020;383(22):e120. https:// doi.org/10.1056/NEJMp2025631

32. Government of Ontario. COVID-19 Guidance: Considerations for Rapid Antigen Point-of-Care Screening.; 2021:7. Accessed March 11, 2021. https:// www.health.gov.on.ca/en/pro/programs/publichealth/coronavirus/docs/ Antigen_Screening_Guidance_2020-12-30.pdf

33. Kubicka W. Extendicare Pilot Findings as Shared with the OLTCA Members via Webinar.; 2021. Accessed March 15, 2021. https:// ontariolongtermcareassociation.app.box.com/s/ ef6qc97z5vq3ymh2no3mbe1ymu42ewzb/file/764626280163

34. Polack FP, Thomas SJ, Kitchin N, et al. Safety and efficacy of the BNT162b2 mRNA COVID-19 vaccine. N Engl J Med. Published online December 10, 2020. https:// doi.org/10.1056/NEJMoa2034577

35. Baden LR, El Sahly HM, Essink B, et al. Efficacy and safety of the mRNA-1273 SARS-CoV-2 vaccine. N Engl J Med. Published online December 30, 2020. https:// doi.org/10.1056/NEJMoa2035389

36. Brown KA, Stall NM, Vanniyasingam T, et al. Early impact of Ontario's COVID-19 vaccine rollout on long-term care home residents and health care workers. Sci Briefs Ont COVID-19 Sci Advis Table. 2021;2(13). https://doi.org/10.47326/ ocsat.2021.02.13.1.0

37. Hall VJ, Foulkes S, Saei A, et al. Effectiveness of BNT162b2 mRNA vaccine against Infection and COVID-19 vaccine voverage in healthcare workers in England, multicentre prospective cohort study (the SIREN study). SSRN. Published online February 22, 2021. https://doi.org/10.2139/ssrn.3790399

38. Amit $S$, Regev-Yochay $G$, Afek A, Kreiss $Y$, Leshem E. Early rate reductions of SARSCoV-2 infection and COVID-19 in BNT162b2 vaccine recipients. The Lancet. Published online February 18, 2021. https://doi.org/10.1016/S0140-6736(21)00448-7

39. Petter E, Mor O, Zuckerman N, et al. Initial real world evidence for lower viral load of individuals who have been vaccinated by BNT162b2. medRxiv. Published online February 8, 2021. https://doi.org/10.1101/2021.02.08.21251329

40. Dagan N, Barda N, Kepten E, et al. BNT162b2 mRNA Covid-19 Vaccine in a Nationwide Mass Vaccination Setting. N Engl J Med. Published online February 24, 2021. https://doi.org/10.1056/NEJMoa2101765 
41. Levine-Tiefenbrun M, Yelin I, Katz $R$, et al. Decreased SARS-CoV-2 viral load following vaccination. medRxiv. Published online February 8, 2021. https:// doi.org/10.1101/2021.02.06.21251283

42. Chodick G, Tene L, Patalon T, et al. The effectiveness of the first dose of BNT162b2 vaccine in reducing SARS-CoV-2 infection 13-24 days after immunization: real-world evidence. medRxiv. Published online January 29, 2021. https:// doi.org/10.1101/2021.01.27.21250612

43. London School of Hygiene and Tropical Medicine. COVID-19 vaccine linked to a reduction in transmission. Published March 12, 2021. Accessed March 12, 2021. https://www.Ishtm.ac.uk/newsevents/news/2021/covid-19-vaccine-linkedreduction-transmission

44. Ontario COVID-19 Science Advisory Table. Ontario dashboard: tracking the third wave. Ontario COVID-19 Science Advisory Table. Published 2021. Accessed March 21, 2021. https://covid19-sciencetable.ca/ontario-dashboard/ 